\title{
A Time-saving method for Transferring Occlusal Vertical Dimension and Centric Relation of Complete Denture to a Full Arch Implant Prosthesis
}

\author{
${ }^{1}$ Fereidoun Parnia, ${ }^{2}$ Elnaz Moslehifard, ${ }^{3}$ Negar Motayagheni, ${ }^{4}$ Alireza Pournasrollah
}

\begin{abstract}
Aim: This clinical report describes a time-saving recording procedure for implant prosthesis.
\end{abstract}

Background: In implant prosthodontics, a few procedures have been suggested to record jaw relationships. Record bases with occlusion rims supported by healing abutments, or screw retained bases are the conventional methods used for mounting definitive implant casts in the articulator.

Technique: This article describes a simple method that enables the clinician to transfer the existing occlusal vertical dimension (OVD) and centric relation (CR) of acceptable complete denture to final fixed prostheses. Advantages and disadvantages of this procedure are also discussed.

Conclusion: The method described in this article reduces the chair time and is comfortable for both the patient and clinician.

Clinical significance: In this article, a chair side technique is presented for articulating the definitive implant casts that maintains the established OVD and CR of the patient's existing complete dentures.

Keywords: Occlusal vertical dimension, Centric relation, Implant.

How to cite this article: Parnia F, Moslehifard E, Motayagheni N, Pournasrollah A. A Time-saving method for Transferring Occlusal Vertical Dimension and Centric Relation of Complete Denture to a Full Arch Implant Prosthesis. J Contemp Dent Pract 2014;15(5):672-675.

Source of support: Nil

Conflict of interest: None

\section{BACKGROUND}

Dental implants have been successfully used for edentulous patients. ${ }^{1,2}$ In order to improve the function, comfort and

\footnotetext{
${ }^{1}$ Associate Professor, ${ }^{2,4}$ Assistant Professor

${ }^{3}$ Postdoctoral Fellow

1,2,4 Department of Prosthodontics, School of Dentistry, Tabriz University of Medical Sciences, Tabriz, Iran

${ }^{3}$ Department of Anesthesiology, Buffalo General Hospital New York, USA

Corresponding Author: Elnaz Moslehifard, Assistant Professor, Department of Prosthodontics, School of Dentistry, Tabriz University of Medical Sciences, Tabriz, Iran e-mail: elnaz_moslehi@yahoo.com
}

satisfaction in edentulous patients, the conventional complete denture may be replaced by an implant supported fixed or removable prosthesis. This is proven as an effective method for treating these patients. The definitive implant casts have been articulated by several techniques at optimal occlusal vertical dimension (OVD) in the correct centric relation (CR) in edentulous patients receiving implants. ${ }^{3-6}$ Record bases with occlusion rims supported by healing abutments, or screw retained bases are the conventional methods used for mounting definitive implant casts in the articulator. ${ }^{3,4}$ However, these approaches demand more patient visits and extra laboratory steps, especially when a favorable relationship is present between the occlusal vertical dimension and centric relation of patient's existing complete denture favorable. Therefore, other methods including the use of duplicated denture with acrylic resin as a custom impression tray, or a duplicated denture using polyvinylsiloxan have been suggested to avoid those disadvantages of the conventional methods. ${ }^{5,6}$ The simultaneous registration of $\mathrm{CR}$ and OVD of the existing denture and relining its intaglio surface on the healing abutments with chair side relining material also has been used. ${ }^{7}$ In this article, a chair side technique is presented for articulating the definitive implant casts that maintains the established OVD and CR of the patient's existing complete dentures. The main advantage of this technique compared to the existing ones is the reduction of time consumed throughout the treatment.

\section{TECHNIQUE}

A 52-year-old woman wearing a removable complete denture for the last 2 years referred to the prosthodontic clinic for implant born fixed prosthodontic reconstruction for the lower jaw. The patient raised a chief complaint to not prefer removable prosthesis. However, she had a problem with poor retention and function during chewing, though she expressed satisfaction with its esthetic. The horizontal and vertical relationships were acceptable. Eight titanium implants (Implantium, Dentinum Co, Korea), $4 \mathrm{~mm}$ wide and $13 \mathrm{~mm}$ long, were placed using a conventional 2-stage protocol (Figs 1 and 2). Patients were given an antibiotic regime using $500 \mathrm{mg}$ amoxycillin, 3 times per day as taken 1 day preoperatively and 5 days postoperatively. Also, 
chlorhexidine mouthwash was given. The implants were allowed to osseointegrate for 4 months. Two weeks after the second-stage surgery, treatments were performed according to the following procedures.

1. Make primary impression of the maxillary prosthesis using alginate (Hydrogum Soft; Zhermark SpA, Bdia Polesine, Italy).

2. Attach eight transfer-type impression copings to the fixture in the mouth.

3. Make mandibular impression with prefabricated closed tray using additional silicone impression material (Panasil Contact Plus, Kettenbach Dental, Eschenburg, Germany).

4. Prepare the custom made open tray (Fig. 3).

5. Join eight transfer type impression copings together using autopolymerized acrylic resin (Pattern Resin; GC Inc, Japan) on the cast and separated by the disk.

6. Join the separated impressions copings together in the mouth using autopolymerized acrylic resin (Fig. 4).

7. Make the final impression using an open-impression tray technique with a polyether impression material (Impregum; 3M ESPE, Seefeld, Germany).

8. Attach implant analogs (fixture analog; Implantium, Dentinum Co, Korea) to the completed impression

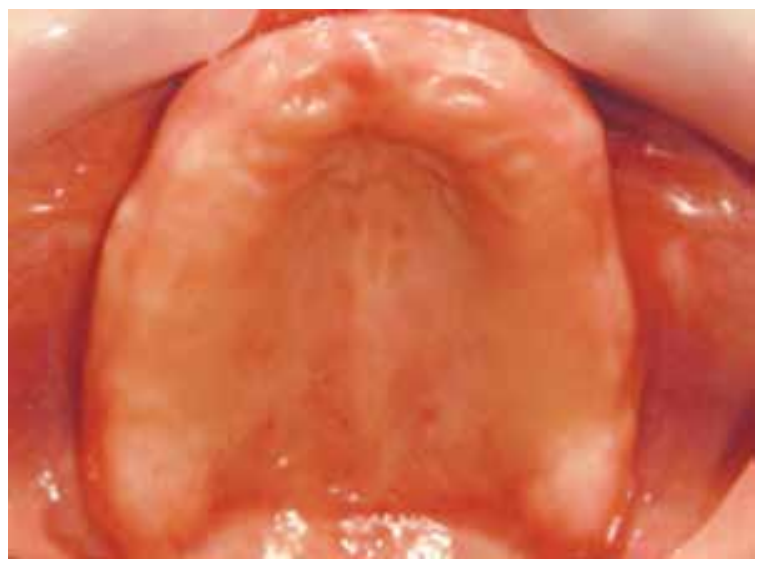

Fig. 1: An occlusal view of maxilla

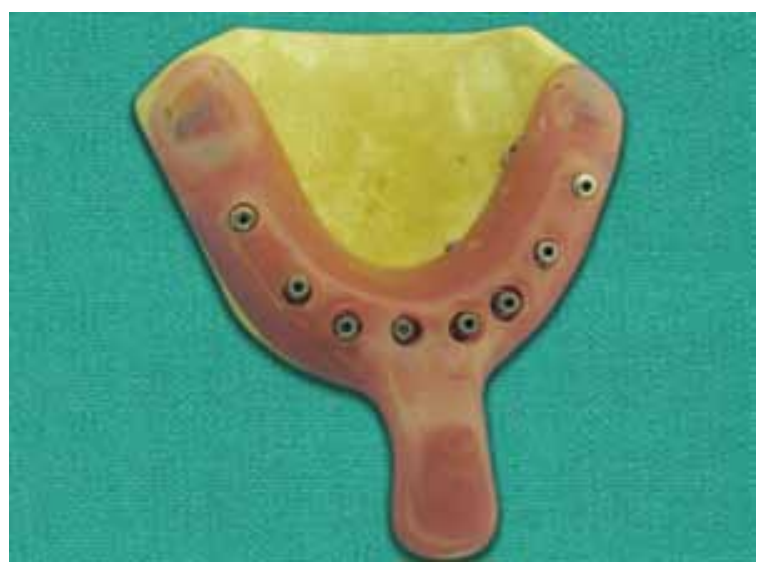

Fig. 3: The image of prepared custom-made open tray and immediately pour type IV dental stone for making impression (Die-Keen; Heraeus Kulzer, South Bend, Ind) to form a definitive cast.

9. Insert four definitive implant level abutments (Dual Abutment; Implantium, Dentinum Co, Korea) in the mouth in which one pair are screwed on each side.

10. Placed denture intraorally and mark abutment position on the intaglio surface of the denture using fit checker (Fit checker II; GC Inc, Japan).

11. Scrape the intaglio surface of the mandibular denture on the four abutments until the denture is seated in the right ultimate position.

12. Drill the holes in a conical manner through the artificial teeth of the mandibular denture over each abutment.

13. The esthetics, phonetics, CR and OVD of the patient's complete denture relieved on the definitive abutments verified.

14. Rigid silicone-type occlusal registration material (occlufast, Zhermark SpA, Bdia Polesine. Italy) injected through the holes around the abutments and an interocclusal $\mathrm{CR}$ record made at the existing established OVD (Figs 5 and 6).

15. Attached the abutments (Dual Abutment, Implantium, Den-tinum Co, Korea) onto the implant analogs of the cast.

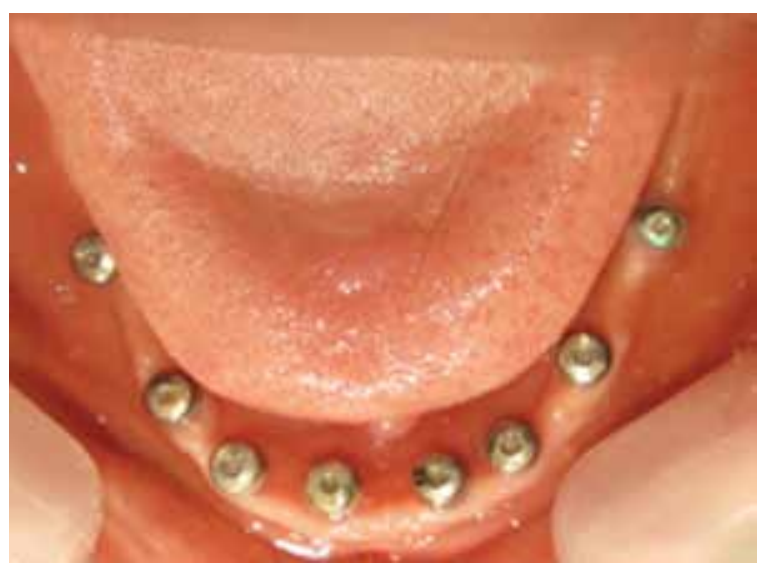

Fig. 2: An occlusal view of mandibule

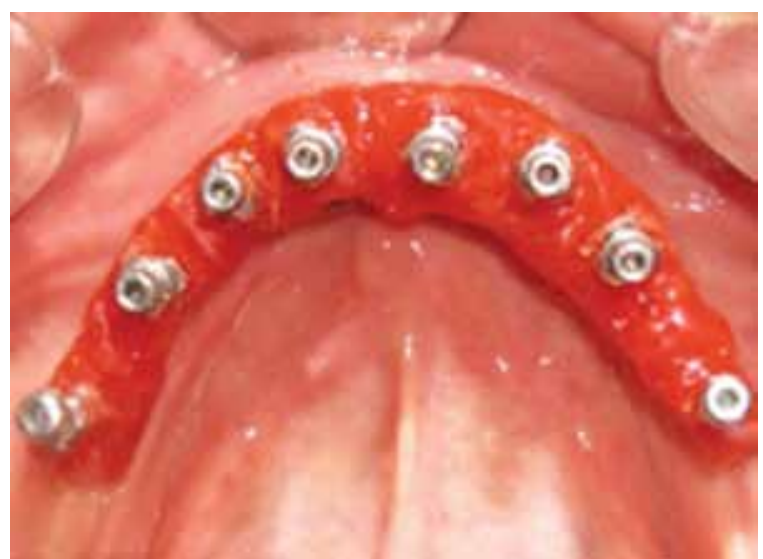

Fig. 4: Joining method of the separated impressions together in the mouth using autopolymerized acrylic resin 


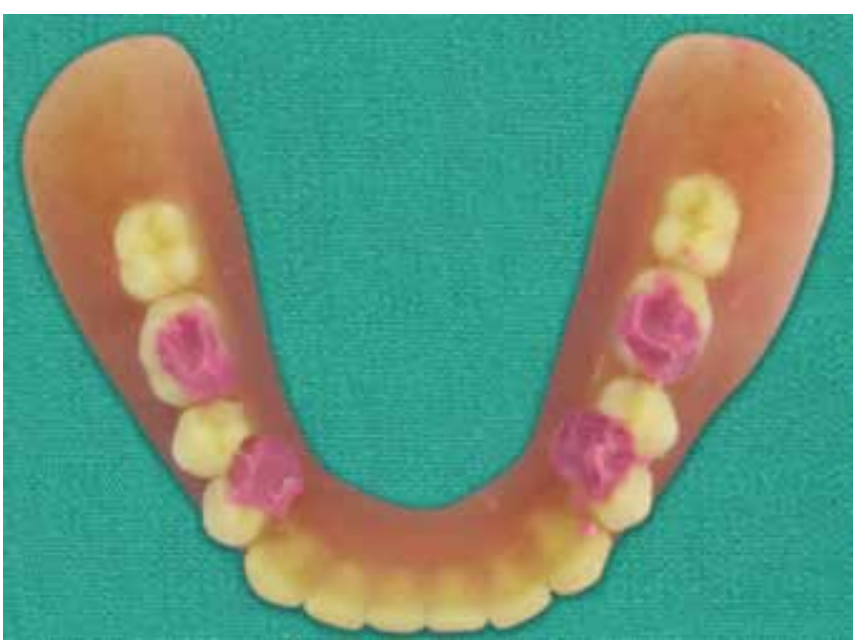

Fig. 5: Interocclusal CR record at the existing established OVD

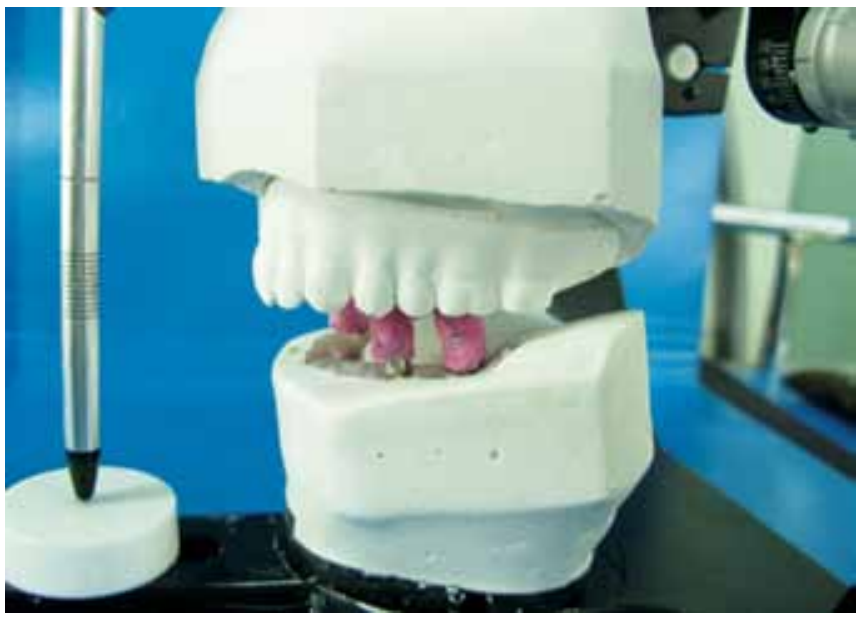

Fig. 7: Articulation of the casts in CR relationship according to the silicon cones

16. Transfer the maxillary denture cast to the articulator with the face bow record (Denar Slidematic facebow; Teledyne Water pik, Fort Collins Co, USA).

17. Transfer the abutments to the mandibular implant cast and place each section of bite registration material over the corresponding abutment.

18. Articulate the mandibular implant cast with the maxillary denture cast, previously mounted on a semi adjustable articulator (Denar Slidematic Articulator; Teledyne Water pik, Fort Collins Co, USA) with the aid of four bite registration material segments (Fig. 7).

19. Attach all of the abutments to the implant analogs in the cast. Make the implant supported fixed partial denture at the established OVD and CR.

20. Plan the follow-up sessions at 1, 2, 3 and 6 months.

\section{DISCUSSION}

This article describes a simple technique to transfer an ideal vertical dimension and centric relation of the patient's previous denture to the definitive cast for fabrication of

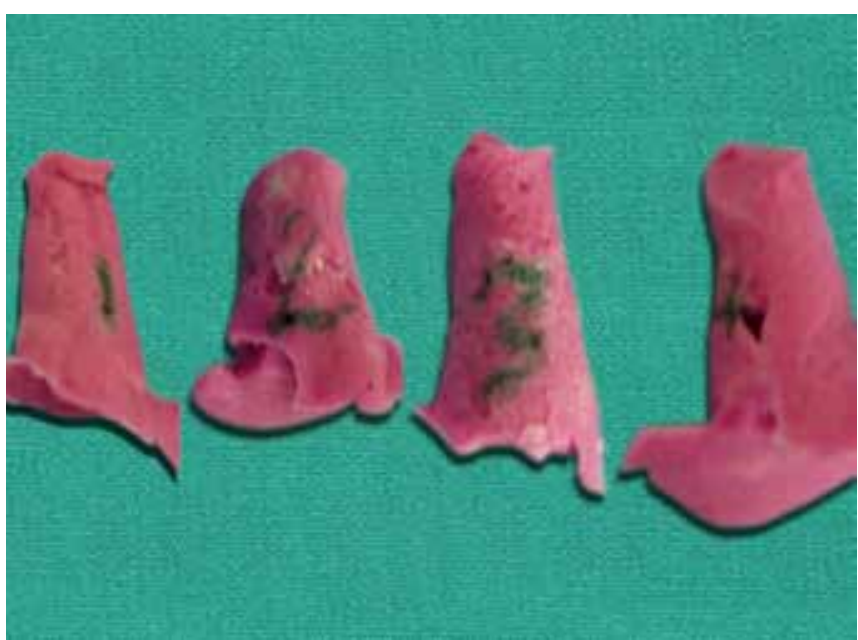

Fig. 6: The silicon cones removed from the denture and marked according the abutment

the implant supported fixed partial denture. The described procedure saves time, because it eliminates the need to fabricate the recording bases and rims. Since, this method reduces the chair time, it provides a significant comfort for both the patient and clinician. The recording process can be carried out using four final abutments and existing complete denture. This method eliminates the need to wax up the final prosthesis before selecting the abutments for the framework fabrication. The existing denture can be utilized as the teeth index. Thus, the laboratory and the chair time for fabrication of the implant supported fixed partial denture can be accelerated. The success of this method depends on a well-established denture. This technique is contraindicated when the existing denture does not meet the ideal esthetic, phonetics, vertical dimension of occlusion and centric relation. When a single arch needs to be restored, the opposing arch can be used as a reference point. However, when restoring both arches, the opposite denture duplicated casts of both arches are mounted in the articulator. Each cast is used as the reference point for the opposing implant working cast at the same VDO. Each working cast is mounted at the same VDO to the opposing cast and the existing CR transferred to the articulator. Also, when adequate implants have not been inserted at all or not been bilaterally inserted, the accuracy of this method decreases. This method can be used to capture the final prosthesis contour to ensure proper fabrication of the implant supported prosthesis by maintaining the position of the prosthesis. This technique is similar to Cranin et $\mathrm{al}^{8}$ and $\mathrm{Misch}^{9}$ that described the use of plastic template to capture the final denture contour. Beumer et $\mathrm{al}^{10}$ and Salinas et $\mathrm{al}^{11}$ used a stone template containing acrylic resin teeth mounted in the articulator to aid in preparation of the metal framework. Using this method, the discrepancy resulted of the shrinkage of the recording bases and distortion of 
the recording materials, such as wax decreases. Selecting a recording material with low discrepancy helps dentists register an accurate $\mathrm{CR}$.

\section{CONCLUSION}

Transferring of an existing OVD and CR to the definitive implant cast for implant supported fixed partial prosthesis has been described. Once a complete removable denture is esthetically accepted by the patient and OVD and CR are favorable, it can be used as a guide for recording as well as for abutment selection. The method described in this article reduces the chair time and is comfortable for both the patient and clinician and also eliminates the need to diagnostic waxup. However, in case of lacking the compatibility between the existing dentures and the ideal esthetic, phonetics, OVD and CR, this method is contraindicated.

\section{CLINICAL SIGNIFICANCE}

In this article, a chair side technique is presented for articulating the definitive implant casts that maintains the established OVD and CR of the patient's existing complete dentures.

\section{REFERENCES}

1. Adell R, Lekholm U, Rockler B, Branemark Pl. A 15-year study of osseointegrated implants in the treatment of the edentulous jaw. Int J Oral Surgery 1981;10(6):387-416.
2. Adell R, Eriksson B, Lekholm U, Branemark Pl, Jem T. Longterm follow-up study of osseointegrated implants in treatment of totally edentulous jaws. Int J Oral Maxillofac Implants 1990; 5(4):347-359.

3. Rungcharassaeng K, Kan JY. Fabricating a stable record base for completely edentulous patients treated with osseointegrated implants using healing abutments. J Prosthet Dent 1999;81(2): 224-227.

4. Chaimattayompol N, Stanescu J, Steinberg J, Vergo TJ JR. Use of across-mounting buccal index to help transfer the spatial relationships of an interim prosthesis to the definitive implant supported prosthesis. J Prosthet Dent 2001;85(5):509-515.

5. Abbo B, Razoog ME. Transfering the existing occlusal vertical dimension using a duplicated denture. J Prosthet Dent 2007; 98(1):68-69.

6. Daher T, Meserkhani PV, Baba NZ, Morgano SM. Time saving method for the fabrication of adefinitive cast for an implant supported prosthesis. J Prosthet Dent 2007;98(1):70-71.

7. Papaspyridakos P, Lal K. Simple technique to transfer occlusal vertical dimension and articulate a definitive implant cast for a full arch implant prosthesis. J Prosthet Dent 2008;100(4): 320-322.

8. Cranin AN, Klien M, Simon A. Atlas of oral implantiology. New York, NY: Thieme Medical Publishers Inc; 1993;213-226.

9. Misch CE. Contemporary implant dentistry. 2nd ed. St. Louis, MO:Mosby Inc 1999;588.

10. Beumer J III, Curtis TA, Marunick MT. Maxillofacial rehabilitation: prosthodontic and surgical considerations. 2nd ed. Brentwood, MO: Ishiyaku Euro America 1996;263.

11. Salinas TJ, Guerra LR, Rogers WA. Aesthetic considerations for maxillary obturators retained by implants. Pract Periodontics Aesthet Dent 1997;9(3):265-276. 